\title{
The Crystal and Molecular Structures of Hydrazine Adducts with Isomeric Pyrazine Dicarboxylic Acids
}

\author{
Wojciech Starosta and Janusz Leciejewicz*
}

Institute of Nuclear Chemistry and Technology, ul. Dorodna 16, 03-195 Warszawa, Poland

\begin{abstract}
The monoclinic unit cell of Bis(hydrazineH) pyrazine-2,3-dicarboxylate contains singly protonated hydrazine cations and doubly deprotonated pyrazine-2,3-dicarboxylate anions. Atoms forming the pyrazine ring are coplanar (r.m.s. $0.0075(1) \AA)$. Carboxylate groups form dihedral angles with the pyrazine ring of $1.7(2)^{\circ}(\mathrm{C} 7 / \mathrm{O} 1 / \mathrm{O} 2)$ and $89.3(2)^{\circ}$ $(\mathrm{C} 8 / \mathrm{O} 3 / \mathrm{O} 4)$. An extended hydrogen bond network is observed in which hydrazine $\mathrm{N}$ atoms act as donors in bonds to nonprotonated carboxylate $\mathrm{O}$ and hetero-ring nitrogen atoms.

The monoclinic structure of hydrazine adduct of pyrazine-2,5-dicarboxylic acid is composed of doubly-deprotonated pyrazine-2,5-dicarboxylate anions and singly-protonated hydrazine cations. The anions have their geometrical centres located at the inversion centres and are planar [r.m.s. 0.0009(1) $\AA$ ]. Both carboxylic groups form dihedral angles of $+11.7(1)^{\circ}$ and $-11.7(1)^{\circ}$ with the pyrazine ring plane. Hydrazine cations acting as donors bridge the anions via weak hydrogen bonds in which carboxylate $\mathrm{O}$ atoms are the acceptors giving rise to molecular layers.

The structure of the adduct with pyrazine-2,6-dicarboxylic acid contains , apart from symmetry independent neutral hydrazine molecules, symmetry independent acid molecules one half of them with protons attached to the carboxylate oxygen atoms as in the parent acid, the second half shows zwitterionic form with proton attached to the hetero-ring nitrogen atom. Carboxylic groups and the pyrazine ring are almost coplanar in both types of acid molecules. The latter are bridged by short hydrogen bonds of 2.435(2) and 2.426(2) Å operating between carboxylate oxygen atoms belonging to adjacent symmetry independent acid molecules, forming layers composed of molecular ribbons. Hydrazine molecules acting as donors link the ribbons by a network of hydrogen bonds.
\end{abstract}

\section{INTRODUCTION}

Hydrazine adducts of organic dicarboxylic acids are interesting due to their potential role in implementing crystal engineering methods. Crystallization from aqueous solutions containing hydrazine made it possible to obtain crystals of organo-metalic compounds which either do not dissolve in water or provide only polycrystalline material. In this way in many cases hydrothermal synthesis can be avoided. The crystal structures of the compounds obtained from hydrazine-containing solutions do not necessarily contain hydrazine molecules (see, for example Ref. [1,2]) but compounds containing in their crystal structures hydrazine molecules have been also observed (see, for example Ref. [3]). In the course of experiments aiming to obtain single crystals from hydrazine-containing solutions, single crystals of hydrazine adducts of diazine carboxylic acids have been found, as for example, an adduct of pyrazine-2,3,5,6-tetracarboxylic acid. Its recently reported triclinic crystal structure [4] contains in the asymmetric unit cell one doubly-protonated hydrazine cation located in an inversion centre, two singlydeprotonated symmetry related pyrazine-2,3,5,6-tetracarboxylate anions and three pairs of symmetry related solvation water molecules. Doubly-protonated hydrazine cations have been recently reported in the crystal structure of a hydrazine adduct of pyrazole-3,5-dicarboxylic acid [5]. Attempting to

*Address correspondence to this author at the Institute of Nuclear Chemistry and Technology, ul. Dorodna 16, 03-195 Warszawa, Poland;

E-mail: jlec@ichtj.waw.pl obtain single crystals of a $\mathrm{Ca}$ (II) complex with pyridazine3,6-dicarboxylate ligand, single crystals of an adduct of the pyridazine-3,6-dicarboxylic acid have been obtained and their crystal structure solved [6]. In all the above adducts hydrazine appears as either singly or doubly-protonated cation and plays an important role in setting up a variety of hydrogen bond networks. For this reason, it was of interest to determine the crystal structures of adducts of all three isomeric pyrazine dicarboxylic acids, since in the course of our studies on $\mathrm{Ca}$ (II) coordination compounds crystallization method from hydrazine containing solutions was frequently adopted and crystals of respective adducts observed. The structures of hydrazine adducts of pyrazine-2,3-dicarboxylic [code name HY(2,3-PZDC)], pyrazine-2,5-dicarboxylic [code name HY(2,5-PZDC)] and pyrazine-2,6-dicarboxylic [code name HY(2,6-PZDC)] acids have been solved and are reported in this paper.

\section{EXPERIMENTAL}

Single crystals of HY(2,3-PZDC) were found during attempts to obtain single crystals of a magnesium complex with pyrazine-2,3-dicarboxylate ligand. When hydrazine was added to control the acidity of the solution, after evaporation to dryness, pale yellow crystals of the adduct were found jointly with the single crystals of the title complex. The same effect was observed in the course of experiments aiming at growing single crystals of a calcium complex with pyrazine2,5-dicarboxylate ligand. Single crystals of HY(2,6-PZDC) were obtained by slow evaporation to dryness at room temperature of an aqueous solution of the title acid after adding 
earlier few drops of $96 \%$ hydrazine solution (ALDRICH). In each case, pale yellow single crystal was separated and used for data collection.

$\mathrm{X}$-ray diffraction data were collected at room temperature using a KUMA-KM4 four circle diffractometer operating in $\omega-2 \theta$ mode. Two standard reflections were monitored every 200 reflections. Unit cell dimensions and standard deviations were obtained by least- squares fit to 25 reflections in the $2 \theta$ range from 15 to $30^{\circ}$. Reflections were processed using profile analysis and corrected for Lorentz and polarization effects. No absorption correction was applied. Final refinement on $\mathrm{F}^{2}$ by full-matrix least squares method was performed on positional parameters of all atoms, anisotropic vibrational parameters of all non $\mathrm{H}$-atoms and isotropic temperature factors of hydrogen atoms. Non - hydrogen atoms were located by direct methods. Hydrogen atoms were found in a difference map and refined. Weighting scheme was used in the form: $\mathrm{W}=1 /\left[\sigma^{2}\left(\mathrm{~F}^{2}{ }_{\mathrm{o}}\right)+(\mathrm{A} * \mathrm{P})^{2}+\mathrm{B} * \mathrm{P}\right]$, where $\mathrm{P}=$ $\left[\mathrm{Max}\left(\mathrm{F}_{\mathrm{o}}^{2}, 0\right)+2 \mathrm{~F}_{\mathrm{c}}{ }^{2}\right] / 3, \mathrm{~A}, \mathrm{~B}$ are the parameters listed in Table 1 with other data collection and refinement parameters. All calculations were performed using SHELX program package [7].

Table 1. Crystal Data and Structure Refinement Details

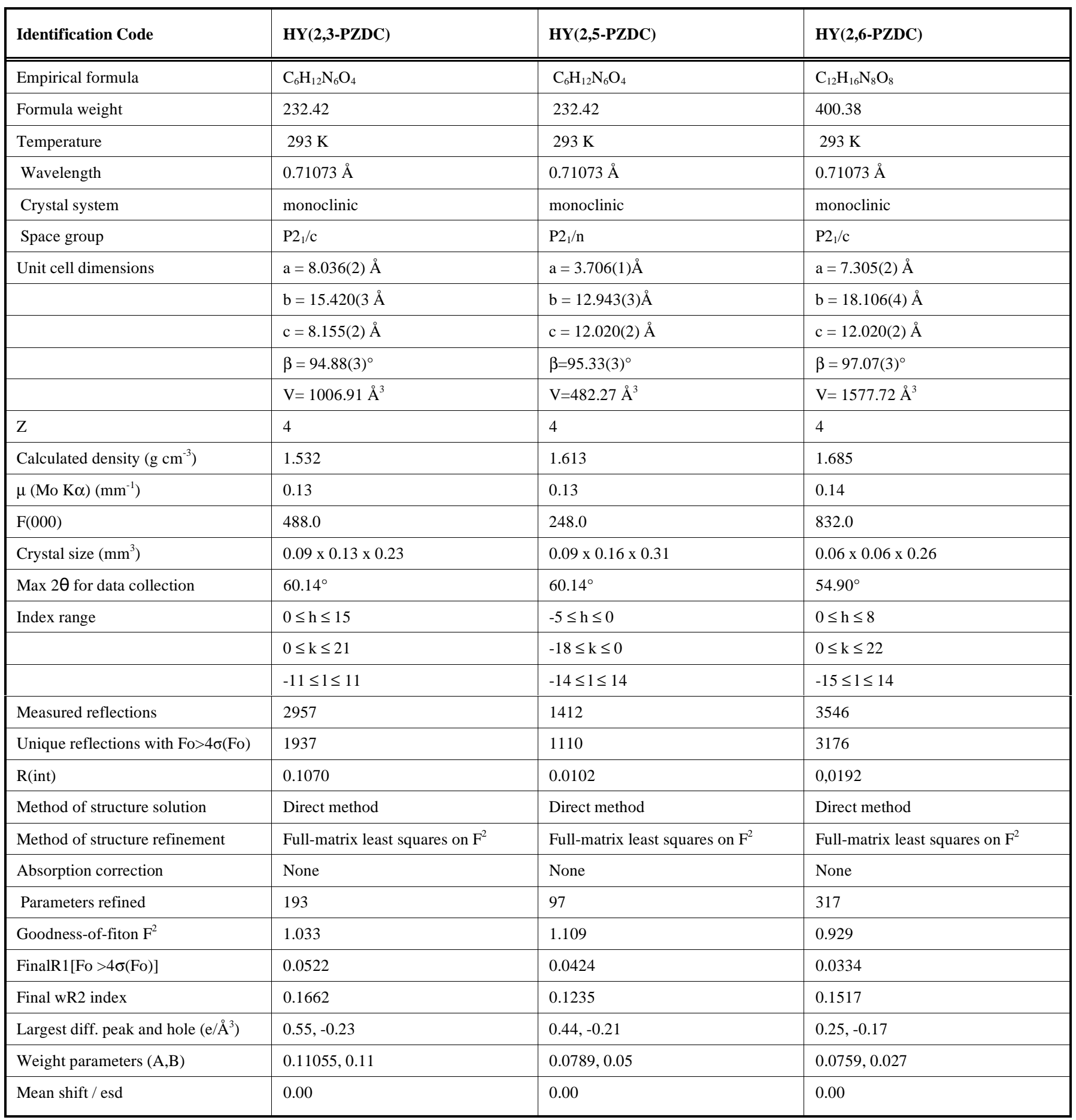




\section{DESCRIPTION OF THE SRUCTURES}

The structure of bis(hydrazineH)pyrazine-2,3-dicarboxylate is composed of singly-protonated hydrazine cations and doubly-deprotonated pyrazine-2,3-dicarboxylate anions. Atoms forming the hydrazine cations belong to two symmetry-independent sites. Consequently, the cation to anion ratio is as 2:1. Fig. (1) shows the atom labeling scheme, whereas Fig. (2). shows the packing diagram with hydrogen bonds marked by broken lines. N-N distances within both protonated hydrazine cations are close to those observed in all up to now reported hydrazine adducts with dicarboxylic acids [4-6]. Atoms forming the pyrazine ring are coplanar [r.m.s. $0.0075(1) \AA]$. The $(\mathrm{C} 7 / \mathrm{O} 1 / \mathrm{O} 2)$ carboxylic group forms with this plane a dihedral angle of $1.7(1)^{\circ}$, whereas for the $(\mathrm{C} 8 / \mathrm{O} 3 / \mathrm{O} 4)$ group the value is $-89.3(2)^{\circ}$. The latter values, as well as the bond distances and bond angles within the anion agree with those observed in the structures of both modifications of pyrazine-2,3-dicarboxylic acid dihydrate $[8,9]$. Hydrazine cations act as donors, carboxylate $\mathrm{O}$ atoms as acceptors in a three-dimensional network of rather weak hydrogen bonds, as indicated by their lengths falling in the range from 2.772(2) to 2,950(2) A. Detailed geometrical parameters are listed in Table $\mathbf{2}$.

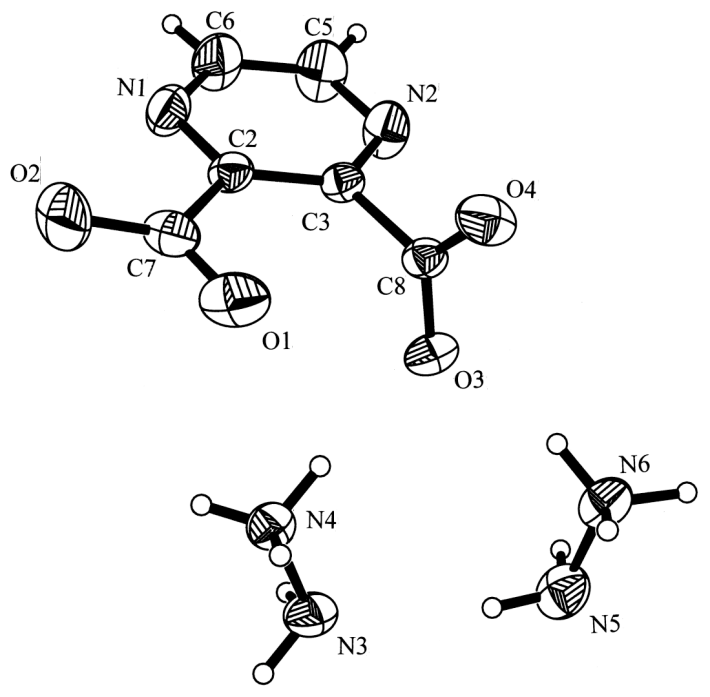

Fig. (1). Bis(hydrazine $H$ ) pyrazine-2,3-dicarboxylate - atom labeling scheme.

The unit cell of hydrazine adduct of pyrazine-2,5dicarboxylate contains four singly-protonated hydrazine cations and two doubly-deprotonated pyrazine-2,5dicarboxylate anions with their geometrical centres at the inversion centres at $1 / 2,0,1 / 2$ and $0,1 / 2,0$. Thus, the cation to anion ratio is as 2:1. Fig. (3) shows a cation and an anion with atom labels. The interatomic distance $\mathrm{N}-\mathrm{N}$ within the cation is 1.440 (2) $\AA$, commonly observed in hydrazine adducts of carboxylic acids. Atoms forming the pyrazine ring

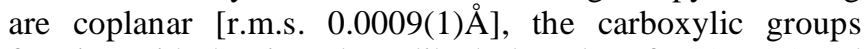
forming with the ring plane dihedral angles of $+11.7(1)^{\circ}$ and $-11.7(1)^{\circ}$. The observed interatomic distances and bond angles do not differ from those reported in the structure of the acid $[10,11]$. The alignment of the cations and anions in the unit cell is shown in Fig. (4). The cations and anions form molecular columns extending along the unit cell $a$ axis with a distance of 3.706(2) A between the centroids of adjacent pyrazine rings. The cations bridge the anions via hydrogen bonds with lengths in the range from 2.755(2) to 2.934(2) A. The cations are the donors and the carboxylate $\mathrm{O}$ and heteroring $\mathrm{N}$ atoms are the acceptors. The geometrical parameters of this hydrogen bond network are collected in Table $\mathbf{3}$.

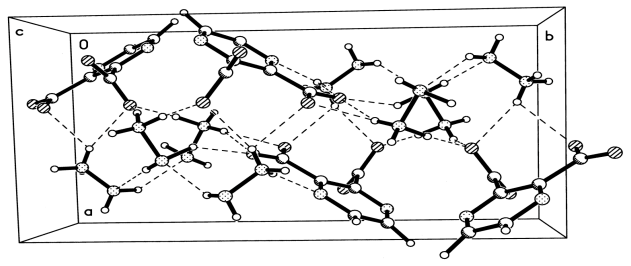

Fig. (2). Packing diagram of bis(hydrazine $H$ ) pyrazine-2,3dicarboxylate. Broken lines show the hydrogen bonds.

Table 2. Geometrical Parameters $\left(\stackrel{\AA}{\circ},^{\circ}\right)$ of Hydrogen Bonds in the Structure of HY(2,3-PZDC)

\begin{tabular}{|c|c|c|c|c|}
\hline & D-H & H...A & D-H...A & D-H-A \\
\hline \hline $\mathrm{N} 3-\mathrm{H} 32 \ldots \mathrm{O} 4^{(\mathrm{i})}$ & $0.89(3)$ & $2.08(3)$ & $2.929(2)$ & $159(2)$ \\
\hline $\mathrm{N} 4-\mathrm{H} 43 \ldots \mathrm{O} 4^{(\mathrm{iii})}$ & $1.06(3)$ & $1.87(3)$ & $2.218(2)$ & $148(2)$ \\
\hline $\mathrm{N} 4-\mathrm{H} 42 \ldots \mathrm{N} 1^{(\mathrm{iv})}$ & $0.88(3)$ & $2.11(3)$ & $2.897(2)$ & $149(2)$ \\
\hline $\mathrm{N} 4-\mathrm{H} 41 \ldots \mathrm{O} 3$ & $0.91(3)$ & $2.05(3)$ & $2.934(2)$ & $165(2)$ \\
\hline $\mathrm{N} 13-\mathrm{H} 16 \ldots \mathrm{O} 1^{(\mathrm{iii})}$ & $0.95(3)$ & $1.99(3)$ & $2.793(2)$ & $142(3)$ \\
\hline $\mathrm{N} 6-\mathrm{H} 62 \ldots \mathrm{O} 3$ & $0.90(3)$ & $1.89(3)$ & $2.772(2)$ & $165(2)$ \\
\hline $\mathrm{N} 6-\mathrm{H} 61 \ldots \mathrm{O} 3^{(\mathrm{v})}$ & $1.08(3)$ & $1.71(3)$ & $2.782(2)$ & $173(3)$ \\
\hline $\mathrm{N} 5-\mathrm{H} 51 \ldots \mathrm{O} 2^{(\mathrm{ii})}$ & $0.97(3)$ & $2.08(3)$ & $2.950(2)$ & $149(2)$ \\
\hline
\end{tabular}

Symmetry code: ${ }^{(\text {(i) }} \mathrm{x}+1, \mathrm{y}, \mathrm{x} ;{ }^{\text {(ii) }}-\mathrm{x}+1, \mathrm{y}+1 / 2,-\mathrm{z}+1 / 2 ;{ }^{\text {(iii) }}=\mathrm{x}+1,-\mathrm{y},-\mathrm{z}+1 ;{ }^{\text {(iv) }}-\mathrm{x}+1,-\mathrm{y},-\mathrm{z} .{ }^{(\mathrm{v})} \mathrm{x},-$ $\mathrm{y}+1 / 2, \mathrm{z}+1 / 2$.
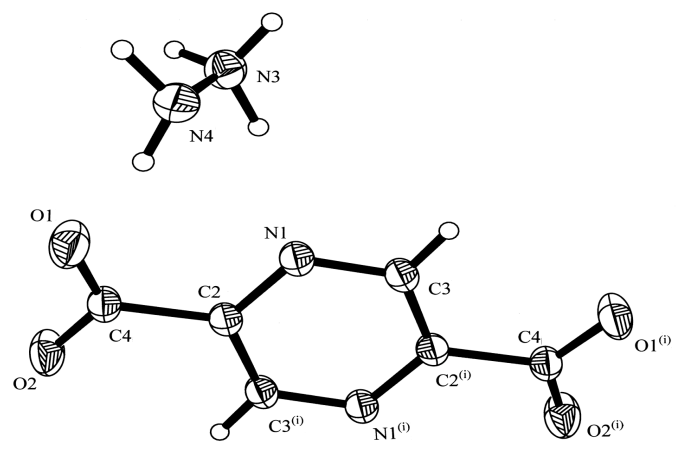

Fig. (3). Hydrazine cation and pyrazine-2,5-dicarbohylate anion with atom numbering scheme. Symmetry code: ${ }^{(i)}-x+1,-y,-z+1$. 


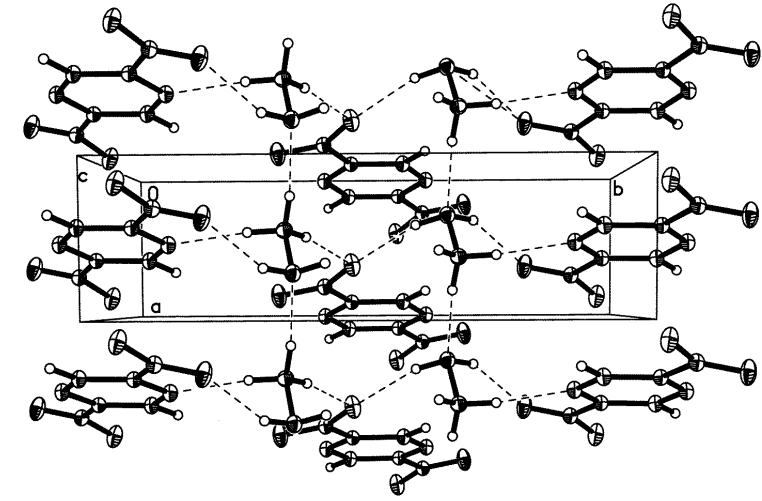

Fig. (4). Packing diagram of hydrazine adduct of pyrazine-2,5dicarboxylic acid.

The structure of the adduct of pyrazine-2,6-dicarboxylic acid contains symmetry independent neutral hydrazine mole-

\section{COMMENT}

Two title adducts exhibit ionic structure with doublydeprotonated pyrazine dicarboxylate anions, in contrast to hydrazine adducts with pyrazine-2,3,5,6-tetracarboxylic and pyridazine-3,6-dicarboxylic acids, in which the anions are singly-deprotonated. The structure of the adduct with pyrazine-2,6-dicarboxylic acid contains neutral hydrazine and acid molecules, a half of them in a zwitterionic form, the latter form being observed for the first time. Zwitterionic forms were earlier reported in the structures of pyridine-2,3dicarboxylic [13] and pyridine-3,4-dicarboxylic acids [14]. The dimensionality of HY(2,3-PZDC) and HY (2,3-PZDC) structures is evidently dependent on the geometry of the anions and the resulting hydrogen bond network. In the structure of the HY(2,3-PZDC) compound, the planes of the carboxylate groups are almost perpendicular each to each other favoring a three-dimensional distribution of the anions and a resulting network of hydrogen bonds. A similar pattern has been reported in the structure of an adduct of pyrazine2,3,5,6-tetracarboxylic acid [4] in which the solvent water molecules also take a significant part in a three-dimensional

Table 3. Geometrical Parameters $\left(\stackrel{\AA}{\mathrm{A}}{ }^{\circ}\right)$ of Hydrogen Bonds in the Structure of HY(2,5-PZDC)

\begin{tabular}{|c|c|c|c|c|}
\hline & D-H & H...A & D-H...A & D-H-A \\
\hline \hline $\mathrm{N} 3-\mathrm{H} 31 \ldots \mathrm{N} 4^{\text {(iv) }}$ & $0.898(18)$ & $2.025(18)$ & $2.9144(15)$ & $170.4(16)$ \\
\hline $\mathrm{N} 3-\mathrm{H} 32 \ldots \mathrm{N} 1$ & $0.95(2)$ & $2.00(2)$ & $2.9339(15)$ & $165.9(17)$ \\
\hline $\mathrm{N} 3-\mathrm{H} 33 \ldots \mathrm{OO} 1^{\text {(ii) }}$ & $0.85(2)$ & $1.92(2)$ & $2.7558(14)$ & $169.2(19)$ \\
\hline $\mathrm{N} 4-\mathrm{H} 41 \ldots \mathrm{OO} 1^{\text {(iii) }}$ & $0.90(2)$ & $2.02(2)$ & $2.8961(14)$ & $164.9(18)$ \\
\hline $\mathrm{N} 4-\mathrm{H} 42 \ldots \mathrm{O} 2$ & $0.892(19)$ & $2.060(19)$ & $152.5(16)$ \\
\hline
\end{tabular}

Symmetry code $-\mathrm{x}+1,-\mathrm{y},-\mathrm{z}+1 ;{ }^{(\mathrm{ii})} \mathrm{x}+1 / 2,-\mathrm{y}+1 / 2, \mathrm{z}-1 / 2 ;{ }^{(\mathrm{iii})}-\mathrm{x}+1 / 2, \mathrm{y}+1 / 2,-\mathrm{z}+3 / 2 ;{ }^{(\mathrm{iv})} \mathrm{x}-1, \mathrm{y}, \mathrm{z}$.

cules and symmetry independent acid molecules with different structures. The structures of one half of acid molecules is the same as in the pyrazine-2,6-dicarboxylic acid [12], the second half shows zwitterionic structure with a proton attached to the hetero-ring $\mathrm{N}$ atom situated outside the carboxylate groups. Fig. (5) shows the atom labeling scheme. The bond lengths and bond angles within the acid molecules do not differ from those reported for the parent acid [12]. The pyrazine ring (1) is planar: r.m.s. $0.0088(2) \AA$, the carboxylic groups (C17/O11/O12) and (C18/O13/O14) make with it dihedral angles of $3.9(1)^{\circ}$ and $5.6(2)^{\circ}$, respectively; the pyrazine ring (2) is also planar: r.m.s. $0.0163(2) \AA$, carboxylic groups (C27/O21/O22) and (C28/O23/O24) form with the ring plane dihedral angles of $2.4(2)^{\circ}$ and $4.6(2)^{\circ}$, respectively. The shortest distance between the centroids of adjacent pyrazine rings is $3.656(2) \AA$ indicating a van der Waals interaction. Linked by strong hydrogen bonds [O22$\mathrm{H} 221 \ldots \mathrm{O} 12^{(\mathrm{i})} 2.433(7) \AA$ and $\mathrm{O} 24-\mathrm{H} 241 \ldots \mathrm{O} 13^{(\mathrm{ii})} 2.428(7)$ $\AA$ ] acid molecules form molecular layers composed of ribbons. Fig. (6) illustrates their packing in the crystal. The ribbons are interconnected by hydrogen bonds in which hydrazine $\mathrm{N}$ atoms act as donors and the carboxylate oxygen atoms are the acceptors. The geometrical parameters of this hydrogen bond network are shown in Table 4.
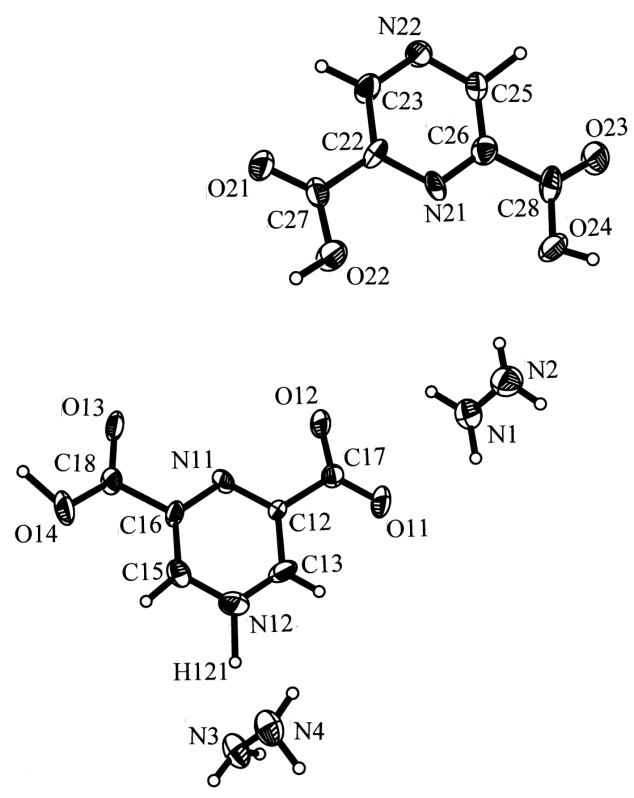

Fig. (5). Molecules constituting the structure of hydrazine adduct of pyrazine-2,6-dicarboxylic acid with atom labeling. 


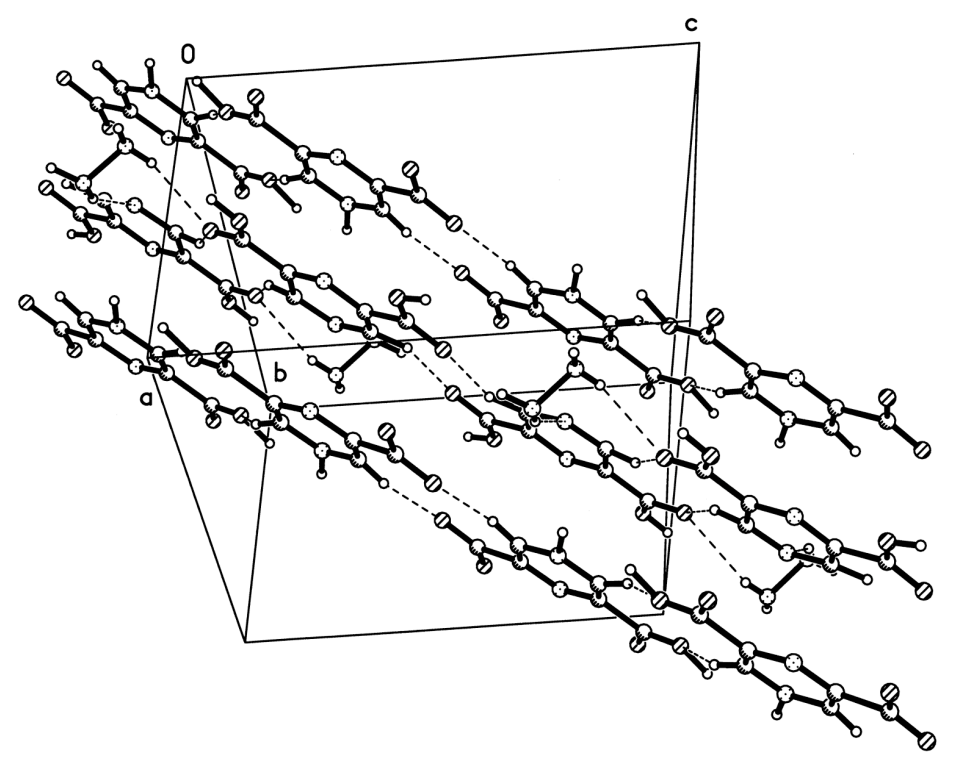

Fig. (6). The alignment of molecules in the unit cell of an hydrazine adduct of pyrazine-2,6-dicarboxylic acid.

Table 4. Geometrical Parameters $\left(\AA{ }^{\circ},{ }^{\circ}\right)$ of Hydrogen Bonds in the Structure of HY(2,6-PZDC)

\begin{tabular}{|c|c|c|c|c|}
\hline & D $-\mathbf{H}$ & H...A & D - H...A & D - H - A \\
\hline \hline $\mathrm{O} 22-\mathrm{H} 221 \ldots \mathrm{O} 12^{\text {(i) }}$ & $0.87(5)$ & $1.57(5)$ & $2.433(7)$ & $176(5)$ \\
\hline $\mathrm{O} 24-\mathrm{H} 241 \ldots \mathrm{O} 13^{(\mathrm{ii})}$ & $0.81(7)$ & $1.65(7)$ & $2.428(7)$ & $160(7)$ \\
\hline $\mathrm{N} 2-\mathrm{H} 21 \ldots \mathrm{O} 12^{\text {(iii) }}$ & $0.85(6)$ & $2.32(6)$ & $2.774(8)$ & $117(5)$ \\
\hline $\mathrm{N} 3-\mathrm{H} 31 \ldots \mathrm{O} 24^{\text {(iv) }}$ & $0.75(7)$ & $2.14(8)$ & $2.876(8)$ & $144(7)$ \\
\hline $\mathrm{N} 3-\mathrm{H} 32 \ldots \mathrm{N} 1^{(\mathrm{v})}$ & $0.94(7)$ & $1.94(7)$ & $2.960(9)$ & $173(5)$ \\
\hline $\mathrm{N} 4-\mathrm{H} 42 . . \mathrm{O} 22^{\text {(iv) }}$ & $0.91(2)$ & $2.19(9)$ & $143(12)$ \\
\hline
\end{tabular}

Symmetry code: ${ }^{(i)} \mathrm{x}+1, \mathrm{y}, \mathrm{z} ;{ }^{(i)} \mathrm{x}, \mathrm{y}, \mathrm{z}-1$ : ${ }^{(\mathrm{iii})} \mathrm{x},-\mathrm{y}+3 / 2, \mathrm{z}-1 / 2$; ${ }^{(\mathrm{iv})}-\mathrm{x}+1,-\mathrm{y}+1,-\mathrm{z}+1 ;{ }^{(\mathrm{v})}-\mathrm{x},-\mathrm{y}+1,-\mathrm{z}+1$.

hydrogen bond network. Since in the 2,5-PZDC anion the planes of the carboxylate groups deviate by only $\pm 11.7(1)^{\circ}$ from the pyrazine ring plane, the anions form molecular columns, similar to the structures of an adduct of pyridazine3,6-dicarboxylic acid [6]. The structure of HY(2,6-PZDC) contains neutral acid molecules in which the carboxylate groups are almost coplanar with pyrazine rings, so that, like in the structure of the parent acid [12], the acid molecules interact via strong hydrogen bonds to show a layer structure.

\section{SUPPLEMENTARY MATERIAL}

Supplementary material can be viewed at www.bentham.org/open/tocryj

\section{REFERENCES}

[1] Premkumar T, Govindarajan S, Starosta S, Leciejewicz J. Diaquatetrakis(pyrazine-2-carboxylato- $\kappa^{2} O, N$ )thorium(IV) trihydrate. Acta Cryst 2005; E61: m98-m100.

[2] Govindarajan S, Premkumar T, Starosta W, Leciejewicz J. Role of hydrazine hydrate in the formation of single crystal of a $\mathrm{Mn}$ (II) complex with pyrazole-3,5-dicarboxylate and water ligands. Inorg Chem Indian J 2007; 2: 1-5.

[3] Gryz M, Starosta W, Leciejewicz J. The crystal and molecular structure of an ionic hydrazinium magnesium(II) complex with pyridazine-3,6-dicarboxylate and water ligands. J Coord Chem 2004; 57: 917-922.

[4] Starosta W, Leciejewicz J. Hydrazinediium bis(2,5,6dicarboxypyrazine-2-carboxylate) hexahydrate. Acta Cryst 2007; E63(Suppl. 1-7): o3734.

[5] Senthil Kumar VS, Premkumar T, Rath NP, Govindarajan S. Polymorphism in hydrozoniun salt of 3,5-pyrazoledicarboxylic acid. Indian J Chem 2007; 46B: 141-147.

[6] Starosta W, Leciejewicz J. Hydrazinediium bis(6carboxypyridazine-3-carboxylate) dihydrate. Acta Cryst 2008; E64(Suppl. 1-3): o46.

[7] Sheldrick GM. A short history of SHELX. Acta Cryst 2008; A64: 112-122.

[8] Takusagawa T, Shimada A. The crystal structure of pyrazine-2,3dicarboxylic acid dehydrate. Chem Lett (Japan) 1973; 1121-1122.

[9] Premkumar T, Govindarajan S, Starosta W, Leciejewicz J. Pyrazine-2,3-dicarboxylic acid. Acta Cryst 2004; E60: o1305o1306.

[10] Ptasiewicz-Bąk H, Leciejewicz J. X-ray diffraction study of the crystal structures of pyrazine-2,5-dicarboxylic acid dihydrate and its magnesium complex. J Coord Chem 1998; 44: 299-309. 
[11] Vishwehrwar P, Nangia A, Lynch VM. Recurrence of carboxylic acid-pyridine supramolecular synthom in the crystal structures of some pyrazinecarboxylic acids. J Org Chem 2002; 67: 556-565.

[12] Ptasiewicz-Bąk H, Leciejewicz J. The crystal structures of pyrazine-2,6-dicarboxylic acid dihydrate and hexaquamagnesium pyrazine-2,6-dicarboxylate. J Coord Chem 2003; 56: 173-180.
[13] Takusagawa T, Hirotsu K, Shimada A. The crystal structure of quinolinic acid. Bull Chem Soc Jpn 1973; 46: 2372-2380.

[14] Takusagawa T, Hirotsu K, Shimada A. The crystal structure of cinchomeronic acid. Bull Chem Soc Jpn 1973; 46: 2669- 2975.

(C) Starosta and Leciejewicz; Licensee Bentham Open.

This is an open access article licensed under the terms of the Creative Commons Attribution Non-Commercial License (http://creativecommons.org/licenses/by-nc/3.0/) which permits unrestricted, non-commercial use, distribution and reproduction in any medium, provided the work is properly cited. 